\title{
COUNTERING COUNTERFEIT BRANDING: \\ AN UNDERSTANDING INCORPORATING MIMESIS AND \\ CULTURAL APPROPRIATION FOR EMERGING MARKETS
}

Tanvir Ahmed, La Trobe University, Australia

Gillian Sullivan Mort, La Trobe University, Australia

Brands designate the most valuable asset that many firms possess, and the associated brand equity is usually the result of years of development efforts. Yet so many brands are becoming increasingly threatened by the worldwide phenomenon of brand counterfeiting (Lai and Zaichkowsky, 1999), whereby counterfeits or imitations of the brands are sold often to unwary consumers as the original. This problem already grasps both developed and developing countries which leads to serious economic, social, and political problem that threaten the lives of unsuspecting consumers, wreaks economic havoc, and weakens consumer confidence in branded products. The global market value of the counterfeit industry is $\$ 600$ billion, with a growth rate of $1700 \%$ over the past 10 years (U.S. Department of State, 2008). The majority of the research on counterfeiting has focused its attention on individual level consumers' motivation towards counterfeit brands applying theories such as "reasoned action" (Woolley and Eining 2006), "planned behavior" (Chang 1998), "expected utility" (Peace et al. 2003), and "ethical decision making" (Wagner and Sanders 2001). However, no research till now has considered counterfeiting through the conceptual lens of culture. This paper responds to persistent calls for research that goes beyond current dominant perspectives and supports a broader view of counterfeit branding. We have introduced a novel approach of cultural appropriation and the theory of mimesis to understand motivations towards counterfeit brands. Our proposed framework suggests that the concept of mimesis can offer an enhanced perspective to understand consumer motivations in regard to products, supply, sell, buy and use of counterfeits. This study incorporates six derived policies and actions (Cooperative, Collective, Strategic, Performative, Conventional and Covert) to educate both consumers and counterfeiters against this negative behaviour.

References available on request 